\title{
VARIANTES ETNO-SOCIALES DEL CASTELLANO EN PUNO
}

Juan Carlos Godenzzi

Universidad Nacional San Antonio Abad,

Cusco.

Un rasgo inherente a las lenguas es su variación, ya sea en el tiempo, el espacio geográfico, el estrato social o la circunstancia del intercambio verbal. El castellano hablado en el Perú en el siglo XVI nó es idéntico al que hablamos hoy; el castellano de Lima y la costa norte difiere del castellano de la sierra o de la selva amazónica. Igualmente, los grupos sociales privilegiados presentan rasgos lingüísticos que los diferencian de los grupos quechuas o aymaras empobrecidos.

El "descubrimiento" de las variantes dialectales y sociolectales de nuestro castellano es un asunto relativamente reciente. Es sólo a partir de 1930 que se comienza tener una conciencia de la situación dialectal del castellano peruano (cf. Carrión 1985: 73). Un primer esfuerzo sistemático de clasificación del castellano en el Perú es el ofrecido por Escobar (1978): se distingue, en primer lugar, el castellano andino (tipo 1) del castellano ribereño (tipo 2) en base al contraste funcional entre $/ \mathrm{ll} \mathrm{y} / \mathrm{y} /$. Enseguida, se hacen ulteriores distinciones en cada uno de los tipos. Así, 


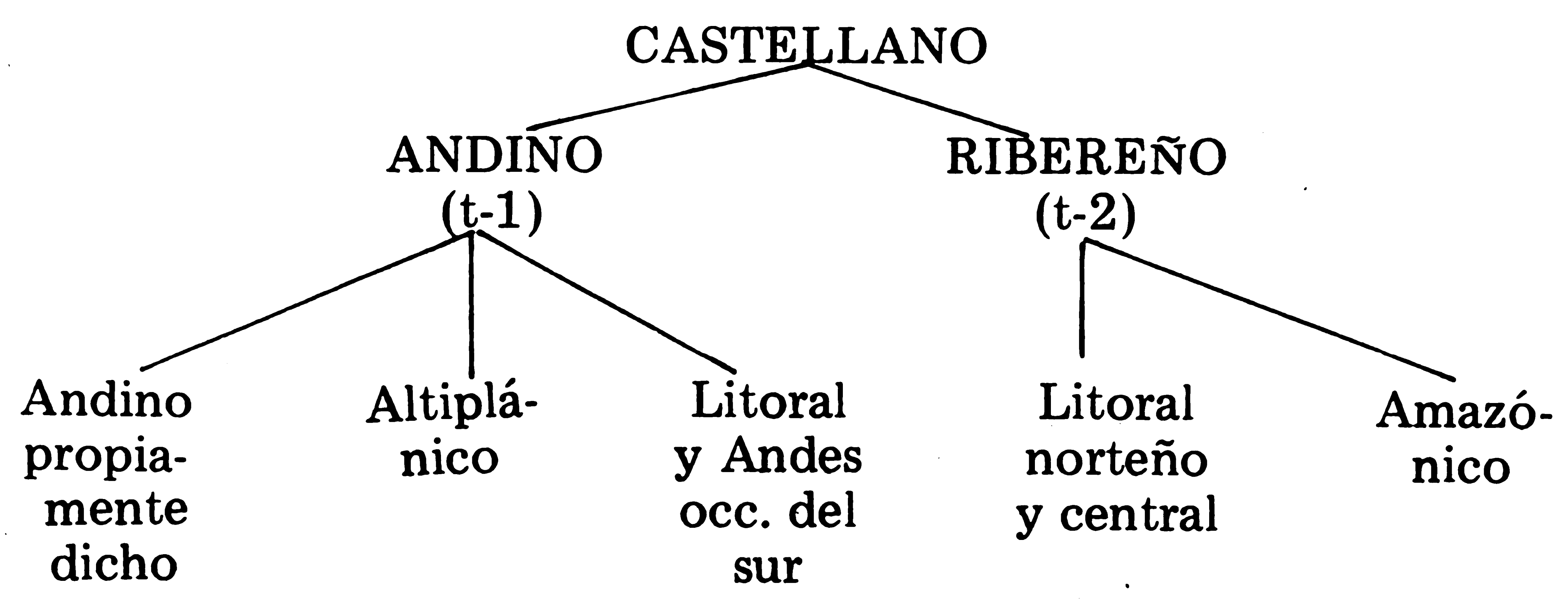

(Elaborado a partir de Escobar 1978: 57-8)

Además de las variaciones percibidas geográficamente, se dan también variaciones etno-sociales que testimonian de las posiciones ocupadas por sus hablantes en un espacio social determinado. La práctica del castellano en el Perú no es ajena a la dinámica social y al ejercicio del poder, tanto a nivel local, regional como nacional (cf. Cerrón-Palomino 1977; Escobar 1978; Godenzzi 1985 y 1986).

En el proceso histórico del castellano en el Perú se puede advertir su evolución en términos sociales. Ya no es la lengua exclusiva de la "nación española" de la Colonia, ni de la "nación criolla" de los inicios de la República. Sobre todo a partir de 1950 el castellano se va convirtiendo cada vez más en la lengua del intercambio a nivel nacional, alcanzando gran parte del interior del país y sus sectores rurales.

En el presente artículo considero las variantes etno-sociales del castellano hablado actualmente en la ciudad de Puno. Para ello tendré en cuenta aspectos fonético-fonológicos, morfo-sintácticos y lexicales.

Debo indicar que la presente exposición es una adaptación de la ponencia que hice en el Seminario Regional de Lingüística Andina y Educación, realizado en Puno, del 23 al 25 de Julio de 1986. Dicha ponencia, a su vez, se basa en mi tesis doctoral (Godenzzi 1985) cuyo material representa unas 70 horas de entrevis- 
tas libres y cuya metodología puede resumirse en los siguientes pasos:

- Construcción de un campo de posiciones sociales (cf. Bourdieu 1980: 113-5), escogiendo para tal efecto los siguientes parámetros: origen étnico, movilidad geográfica, nivel económico, capital escolar. Así, cada uno de los hablantes encuentra su lugar al interior de este campo.

- Inventario de las soluciones lingüisticas, a partir de algu: nas unidades de lengua. Se sigue una orientación onomasiológica; es decir, un trayecto que parte de categorías semántico-conceptuales y que llega a las formas lingüísticas que las expresan.

- Distribución de las soluciones lingüísticas al interior del campo de posiciones sociales, permitiéndose así la visualización, el análisis y la interpretación de las variantes.

\section{VARIANTES ETNO-SOCIALES}

Para cada unidad lingüística estudiada proveo: las soluciones practicadas, su distribución etno-social y algunos comentarios sociolingüísticos.

El yeísmo

Los hablantes practican alguna de estas soluciones:

a: $11>$ y (yeísmo). Ej: castellano-[kasteyáno]

$\mathrm{b}: \mathrm{ll} \neq \mathrm{y} \quad($ no-yeismo $)$. Ej: castellano-[kastelláno]

c. ll $>$ y (alternancia de yeísmo y no-yeísmo)

$11 \neq y$

La solución $b$ es fuertemente practicada por los nacidos en Puno. Sin embargo, hay puneños que recurren a la solución $c$ : se trata de puneños de tradición urbana, que han pasado buenos períodos en Lima o la zona costeña y que tienen buenos años de escolaridad. La solución $a$ es practicada por los limeños y costeños 
residentes en Puno: a pesar de sus largos años de residencia en la región, no abandonan su yeísmo.

El yeísmo va asociado con una clase de prestigio: ser "limeño" o "costeño" por oposición a "serrano", que a menudo posee una connotación peyorativa. Pero el no-yeísmo tiene también otra clase de prestigio, si bien subordinado: ser "autóctono", "neto", "verdadero puneño". Estas dos clases de prestigio pueden eventualmente entrar en conflicto, expresando desconfianzas entre los puneños y los "venidos de fuera". Me parece que la oposición yeísmo / no-yeísmo puede de alguna manera expresar la identificación con un espacio regional, pudiendo también ser erigida en eventual frontera de dos grupos en tensión.

\section{/y/ en posición intervocálica}

Se tienen estas soluciones:

$a:[y]$ Ej: proyecto - [proyékto]; mayo - [máyo]

$b$ : a veces $[\mathrm{y}]$, a veces $[\hat{\mathrm{y}}]$. Ej: ayudar - [ayudár]

$$
\begin{aligned}
& \text { destruye }- \text { [destrúŷe] } \\
& \text { pienso yo }- \text { [pjensoyó] } \\
& \text { hasta Yunguyo - [astaŷungú- } \\
& \text { yo] }
\end{aligned}
$$

La solución a puede ser encontrada en todas las posiciones. La solución $b$, en cambio, puede ser encontrada especialmente en los puneños de tradición urbana, que han residido permanentemente en Puno y que pertenecen a los grupos menos pobres.

Si se considera la edad y el sexo de los puneños que practican la solución $b$, hay que decir que se trata mayormente de la generación más joven (menores de 20 años) y del sexo femenino. Me parece que en una sociedad fuertemente marcada por el mestizaje se pueden encontrar ciertas "sensibilidades" muy predispuestas a distinguirse por medios más o menos sutiles. La pronunciación afectada del fonema /y/ en posición intervocálica es, a mi parecer, un medio disponible para la nueva generación que ocupa ciertas posiciones a fin de distinguirse socialmente. Mientras que ciertos puneños recurrían al yeísmo en su afán de ascen- 
so social, así también otros recurren a la pronunciación [ŷ] en posición intervocálica buscando un beneficio social.

\section{El artículo}

Considerando el uso de los artículos un / el, voy a distinguir tres tipos de soluciones:

a: soluciones donde predomina la no-omisión de los artículos.

b: soluciones donde se encuentra una alternancia de omisiones y no-omisiones de los artículos.

c: soluciones donde predomina la omisión de artículos.

Las soluciones que corresponden al tipo $a$ están presentes en todas las posiciones. Las soluciones tipo $b$ aparecen en algunos puneños de tradición urbana y que no han tenido largos períodos fuera de Puno, así como en puneños de origen quechua o aymara. Las soluciones tipo $c$ aparecen en el grupo más desfavorecido económicamente y el más marcado por su origen quechua-aymara.

¿A qué se debe la omisión de artículos en las soluciones tipo $b$ y $c$ ? Además de factores propios al sistema de artículo español, me parece que juega un rol importante el hecho de haber en Puno una importante población que habla el quechua o el aymara como primera lengua, considerando que tales lenguas no tienen la categoría de artículo. Pasar cle una lengua donde no hay artículos a otra donde sí los hay, puede implicar una etapa intermedia donde a veces se explicitan y a veces se omiten los artículos. Una vez que las circunstancias del aprendizaje del castellano a partir del quechua o el aymara han hecho aparecer las frecuentes omisiones de los artículos, es posible que estas soluciones sean adoptadas como formas alternativas más o menos duraderas. La distribución de estos tipos de soluciones nos revela "rupturas" en la continuidad del espacio social, según la influencia quechua-aymara sea mayor o menor. Así, el uso u omisión del artículo es un ejemplo de cómo el factor quechua-aymara condiciona la adquisición del castellano, introduciendo eventualmente soluciones lingüísticas alternativas y pudiendo servir de base para una fina discriminación etno-social. 


\section{Adjetivación de tercera persona}

A fin de evitar la ambigüedad, los hablantes recurren a una de estas soluciones:

a: no se utiliza el adjetivo personal su; más bien, se recurre a la forma analítica: [de $+3 \mathrm{ra}$. pers.] "las crías de la parihuana"

b: se utiliza el adjetivo personal su y se añade la forma analítica. Es decir: [su + de + 3ra. pers.] "en la casa de sus papás de Fredy"

La solución $a$ está presente en todas las posiciones. La solución $b$ está particularmente presente en puneños de tradición urbana y que no han salido de la región y en puneños de origen rural quechua-aymara; pero también puede ser encontrada, aunque en menor escala, en los otros grupos.

Me parece que la razón de la existencia de la solución $b$ hay que buscarla en la indeterminación propia a la naturaleza de la cateogría de 3ra. persona (hecho considerado universal) y en la ambigüedad congenital de la forma su (hecho particular al sistema español). Cuando el hablante recurre a la forma $s u$, puede advertir que la referencia a la persona queda en la indeterminación, inclinándose así a añadir el giro analítico. Esta explicación puede permitir comprender mejor la constante posibilidad de la solución $b$ en la lengua española: en el español hablado de Puno hoy, pero también en el español antiguo.

La solución $b$, explicable al interior del sistema castellano, encuentra un uso frecuente en Puno, caracterizando al castellano de la región. Pero quienes utilizan más esta forma regional son precisamente los puneños que no han tenido mayores contactos extra-regionales y que pertenecen a los grupos con menor capital económico-escolar. Esto nos revela, pues, que en ciertos grupos de puneños se da una convergencia de la regional y popular: la solución $b$ sería una de sus expresiones. 


\section{Pronombres de Objeto Directo e Indirecto}

En lo que se refiere a las formas utilizadas para sustituir tanto al Objeto Directo (OD) como al Objeto Indirecto (OI), se han identificado las siguientes soluciones:

$$
\begin{aligned}
& \text { a: lo(s), la(s) para el OD; le(s) para el OI. } \\
& \text { b: }\{\text { lo(s), le(s)\}, }\{\text { la(s), le(s)] para el OD; le(s) para el OI. } \\
& \text { c: }\{\text { lo, lo(s)] para el OD; }[\text { le, le(s)] para el OI. }
\end{aligned}
$$

Los "venidos de fuera" recurren a la solución $a$. Los puneños de origen citadino y que tienen el castellano como primera lengua practican sea la solución $a$ sea la $b$. Los puneños de origen campesino y que tienen el quechua o el aymara como primera lengua practican sobre todo las soluciones $c$ y $b \mathrm{y}$, en mucho menor medida, la $a$.

Me parece que la solución $c$, practicada por puneños cuya primera lengua es el quechua o aymara, tiende, negligiendo las marcas de género y número, hacia la constitución de un lo invariable y polivalente. Esta economía en las marcas de género y número encuentra su explicación más inmediata en el sustrato quechua-aymara, lenguas en las que la concordancia es puesta en obra de un modo facultativo. La distribución social de estas soluciones nos muestra una triparlición en la continuidad: los "venidos de fuera", los puneños de tradición urbana y los puneños de origen rural quechua-aymara.

En cuanto a la utilización redundante del pronombre de OD, he considerado dos contextos diferentes:

Contexto 1: el pronombre-OD aparece antes que el Sintagma Nominal-OD. "lo dominan la lengua".

Contexto 2: el Sintagma Nominal-OD aparece antes que el pronombre-OD: "el castellano lo dominan bien".

Así, tendríamos estos tres tipos de soluciones: 
a: débil redundancia en 1 , fuerte en 2 .

b: redundancia considerable en 1 , fuerte en 2 .

c: redundancia considerable en 1 y en 2 .

Los venidos de Lima o la costa norte practican sólo la solución tipo $a$. Los puneños de tradición urbana que han pasado un buen tiempo fuera de Puno y los que no han pasado un tiempo considerable fuera de Puno y que pertenecen a la categoría económica más favorecida practican la solución tipo $a \mathrm{y}$, en menor medida, la $b$. Los puneños de tradición rural quechua-aymara que son al mismo tiempo los menos fávorecidos económicamente, practican las soluciones ya sea del tipo $a, b$ o $c$.

El orden libre de las palabras y la frecuente ausencia de pronombres sujeto son rasgos propios del sistema español. Esto motiva que se recurra a menudo a la redundancia; pero ésta se la hace según preferencias socio-diferenciales: los grupos con mayor. capital económico-escolar y mayor capital etno-cultural prefieren la redundancia en el contexto 2 e intentan evitarla en el contexto 1. Los grupos menos "favorecidos" tienden a recurrir a la redundancia de un modo más libre y homogéneo, tanto en el contexto 1 como en el 2 . Esto nos revela actitudes diferentes ante la lengua: sea una actitud más influida por criterios formales de corrección a menudo preocupada por el beneficio social, sea una actitud más libre ante las posibilidades de la lengua misma.

\section{Tiempo verbal}

Las lenguas se las arreglan para encontrar formas que le permitan a uno situarse con precisión en el tiempo. Una de las soluciones dadas por el castellano es la que distingue tres épocas: futuro, pasado y presente.

Para expresar la categoría conceptual /FUTURO NO INMEDIATO/ el castellano ofrece, entre otras, estas formas:

\section{1: cantaré \\ 2: voy a cantar}

Las soluciones dadas por los hablantes combinan diferentemente estas formas: 
a: presencia importante de las formas 1 y 2 .

b: presencia predominante de la forma 2 .

c: presencia exclusiva de la forma 2 .

Los "venidos de fuera" y los puneños de tradición citadina que pertenecen a los grupos económicamente más favorecidos practican fuertemente las soluciones $a$ y $b$. Los puneños de tradición citadina sin haber salido de Puno recurren sobre todo a la solución $b$. Los puneños de origen quechua-aymara y que pertenecen a los grupos económicamente menos favorecidos recurren fuertemente a las soluciones $b$ y $c$.

Para formular la categoría conceptual /PRETERITO/, el castellano nos ofrece estas formas:

\section{1: canté \\ 2: he cantado}

Las soluciones dadas por los hablantes combinan estas dos formas:

a: presencia predominante de la forma 1 .

b: presencia importante de las formas 1 y 2 .

c: presencia predominante de la forma 2.

Los "venidos de fuera" y los puneños de tradición urbana que han pasado largo tiempo fuera de Puno y con mayor capital económico-escolar practican la solución $a$. Los otros puneños de tradición urbana practican fuertemente las soluciones $a$ y $b$. Los puneños de tradición rural quechua-aymara practican fuertemente las soluciones $b$ y $c$.

Para formular la categoría /PRESENTE ACTUALIZADO/, el castellano dispone de estas dos formas posibles:

1: canto

2: estoy cantando

Las soluciones de nuestros hablantes combinan estas formas: 
a: presencia predominante de la forma 1 .

$\mathrm{b}$ : fuerte presencia de las formas 1 y 2 .

c: presencia predominante de la forma 2 .

Los "venidos de fuera" practican la solución $b \mathrm{y}$, en menor medida, la solución $a$. Los puneños de tradición citadina practican sobre todo la solución $b \mathrm{y}$, en menor medida, las soluciones $a$ y $c$. Los puneños de tradición quechua-aymara practican fuertemente las soluciones $b$ y $c$; en mucha menor medida, la solución $a$.

Advertimos que las formas 1 son formas simples y que las formas 2 son perifrásticas. Igualmente, que se da un conflicto entre formas simples / formas perifrásticas. Los resultados obtenidos indican algunas tendencias:

- las formas perifrásticas ganan mucho más terreno en los grupos menos favorecidos.

- se da un conflicto importante entre las formas simples y las perifrásticas en los grupos medios y en los grupos que tienen más capital etno-cultural y económico-escolar.

- la proporción más grande las soluciones que contienen las formas simples se encuentra en los grupos que tienen más capital etno-cultural y económico-escolar.

Subrayo la tendencia que muestra una preferencia o gusto de los sectores populares en favor de las formas perifrásticas.

Elementos de relación

Al utilizar los deícticos, algunos hablantes recurren al uso redundante del relator "en" (en aqui). Distingamos tres soluciones:

a: ausencia de formas redundantes

b: presencia no negligible de formas redundantes

c: presencia considerable de formas redundantes.

Las formas redundantes contenidas en las soluciones $b$ y $c$ no están estrechamente localizadas. Su extensión alcanza, al me- 
nos en parte, a todos los grupos, excepto a los venidos de Lima o la costa norte. El uso más frecuente de la solución $c$ se da en los puneños de tradición quechua-aymara.

En lo que se refiere al relator "de", hemos encontrado en Puno utilizaciones que nos parecen curiosas ("otro casa ha regalado de almuercito, otro casa ha regalado de desayunito...", "...no creo que haya de trabajos", "no sabía ni hablar de castellano..."). Distingamos tres soluciones:

\section{a: ausencia de formas "curiosas" \\ b: presencia no negligible de formas "curiosas" \\ c: presencia considerable de formas "curiosas"}

Las formas curiosas contenidas en las soluciones $b$ y $c$ tienen una amplia extensión, pudiendo alcanzar a todos los grupos. Sin embargo, el uso más frecuente de dichas formas (solución $c$ ) se da en los puneños de tradición quechua-aymara y en los puneños de tradición citadina que no han pasado períodos largos fuera de Puno y que no pertenecen a la categoría económica más favorecida. El uso menos frecuente de dichas formas se da en los grupos de los "venidos de fuera".

¿Cómo explicar este uso especial de los relatores "en" y "de"? Propongo hacerlo recurriendo a la situación de crisis que puede derivarse del proceso de adquisición del castellano a partir del quechua o del aymara, sobre todo si se da en condiciones desfavorables. En el caso del "en" redundante se trata de una interferencia: en quechua y en aymara el deíctico está especificado por un elemento de relación (los sufijos $-p i \mathrm{y}-n a$, respectivamente, kaypi, akana). Este hábito de explicitar el deíctico por medio de un relator puede continuar cuando se comienza a hablar castellano como segunda lengua: se tenderá a especificar el deíctico aqui con el relator en (en $a q u i)$. En lo que se refiere a las soluciones curiosas de "de", se trataría más bien de la dificultad para llegar al uso "acertado" de este relator, teniendo en cuenta la situación de trastornos que comporta el aprendizaje, pero también el hecho de que la utilización "superflua" de dicho relator se presenta en ciertos giros del español (recordar de, dar de palos, hacerse de la vista gorda...; digo de que, pienso de que...). 
La distribución social de estas soluciones nos muestra su presencia más frecuente, aunque no exclusiva, en los grupos de puneños con fuerte tradición quechua-aymara, expresando los esfuerzos de afirmación social por parte de agentes o grupos de agentes que han hecho su ingreso en el ámbito social urbano de Puno.

\section{La concordancịa}

En cuanto se refiere al género, propongo distinguir estos tipos de soluciones:

a: soluciones donde se encuentra una fuerte presencia de concordancias. Eventualmente se dan conflictos semántico-sintácticos.

b: soluciones donde se encuentra una presencia no negligible de no concordancia. En muchos casos se busca economizar las marcas redundantes de género.

c: soluciones donde se encuentra una fuerte presencia de no cóncordancia. Además de conflictos semántico-sintácticos y de reducciones de marcas redundantes de género, se dan dificultades que tienen que ver con una alteración en los constituyentes del sustantivo.

Las soluciones tipo $a$ están presentes sobre todo en los "venidos de fuera" y en los puneños de tradición urbana. Las soluciones tipo $b$ están presentes sobre todo en los puneños de tràdición urbana. Las soluciones tipo $c$ están fuertemente presentes en el grupo de puneños de origen quechua-aymara.

En lo que concierne al número, propongo distinguir también estos tipos de soluciones:

a: soluciones donde se encuentra una fuerte presencia de concordancias. Eventualmente se dan conflictos semántico-sintácticos.

b: soluciones donde se encuentra una presencia no negligible de no concordancia. En muchos casos se busca economizar las marcas redundantes de número.

c: soluciones donde se encuentra una fuerte presencia de no concordancia. Se dan, además, dificultades debidas a una práctica más libre que afecta el comportamiento habitual 
del sustantivo (singular o plural según el contexto discursivo) y a una transgresión más o menos sistemática al principio de concordancia entre la Base y el Predicado.

Las soluciones tipo $a$ están presentes sobre todo en los "venidos de fuera" y en los puneños de tradición urbana. Las soluciones tipo $b$ están presentes sobre todo en los puneños de tradición urbana. Las soluciones tipo $c$ están presentes sobre todo en el grupo de puneños de origen quechua-aymara.

Entre los factores que resisten al principio de la concordancia de género y número, podemos señalar:

- el proceso de adquisición del castellano a partir del quechua o el aymara, lenguas donde el sustantivo no lleva género gramatical y donde el sustantivo y sus determinantes no concuerdan obligatoriamènte en género y número.

- la concordancia excesiva del castellano. Un principio de economía se levanta contra este exceso. español.

- el carácter arbitrario del género gramatical del sustantivo

Eventualmente estos factores pueden ser convergentes.

A pesar de la influencia escolar que insiste en una concordancia fuertemente marcada por el criterio sintáctico, se puede decir que, en general, el castellano de los puneños, sobre todo el de los sectores menos favorecidos, se guía de un modo significativo por el principio de economía que reduce las marcas excesivas de género y número. Esta práctica bien arraigada revela un sentido de sobriedad, así como un cierto buen gusto al evitar las soluciones excesivamente redundantes.

Las transgresiones presentadas por las soluciones que expresan incertidumbre en la atribución de una marca de género al sustantivo permiten identificar a sus autores como hablantes cuya primera lengua no es el castellano, sino el quechua o el aymara. ¿Puede esta práctica cuestionar al sustantivo español encaminán- 
dolo hacia una simplificación de sus constituyentes? Quitar el género gramatical al sustantivo significaría liberarlo de un peso no necesario. Pero me parece remota la efectividad de esta virtual influencia, considerando que estas soluciones se dan predominantemente en los grupos más desfavorecidos. Más bien, estos indicios se convierten muchas veces en medio de desprecio y discriminación. Las soluciones más sobrias y elegantes no son siempre las que gozan de mayor consideración social.

\section{Elecciones léxicas}

A partir de un /CONCEPTO/ reconstruido teóricamente se pueden inventariar las diferentes soluciones que los hablantes han encontrado. Así, por ejemplo, para expresar el concepto /NIÑO RECIEN NACIDO/ se tienen, entre otras, estas soluciones:

\section{b: bebe \\ w: wawa, wawito}

La solución $b$ puede ser encontrada en todos los grupos. En cambio, la solución $w$ sólo puede ser encontrada en los grupos de los nacidos en Puno.

Para expresar el concepto /HOMBRE ADULTO/, dentro del contexto de la vida social, se tienen, entre otras, estas soluciones:
s: señor
$\mathrm{h}$ : hombre
c: caballero

Los grupos con mayor capital económico-escolar prefieren la solución $s$, aunque recurren también, en menor medida, a la solución $h$. Los grupos con menor capital económico-escolar prefieren las soluciones $c$ y $h$, aunque recurren también, con poca frecuencia, a la solución $s$.

El hablante es el único responsable en la elección de sus designaciones léxicas; pero ahí intervienen diversos factores condicionantes como, por ejemplo, el de su posición social. Es así como ciertas soluciones léxicas están asociadas a determinadas con- 
notaciones sociales: wawa puede connotar el "origen regional puneño" de su productor; caballero puede connotar el "nivel económico medio o bajo" de su productor.

\section{BALANCE}

Los resultados generales nos muestran que cada unidad lingüística estudiada presenta su propia distribución en el campo social de posiciones. Cada solución o tipo de solución asegura cierta cohesión de grupo, pero al mismo tiempo establece sutiles rupturas y exclusiones. El conjunto de las variantes etno-sociales se nos presenta como un juego de solidaridades que se hacen y se deshacen incesantemente. Se trata siempre de divisiones en la continuidad, donde las fronteras no son siempre bien definidas. En un orden de jerarquía, sin embargo, la variable étnica juega un papel importante: la división más notable para el caso de la ciudad de Puno es la tripartición entre los "venidos de fuera", los puneños mistis de tradición urbana y los puneños de origen quechua-aymara. En cada uno de estos grupos se configuran ciertos hábitos que tienen una relativa estabilidad y que sirven de base para estrategias de afirmación de la identidad o del ascenso social.

\section{PERSPECTIVAS}

\section{Estudios complementarios}

Nuestra exposición se ha centrado sobre las variantes lingüísticas en relación con las posiciones socio-culturales. Pero otros estudios podrían ser vislumbrados: estudios sobre la percepción que los hablantes tienen del mundo social; estudios de las creencias y opiniones que los hablantes tienen de su lengua. Esto también interesa a la lingüística, puesto que influye en el funcionamiento de la lengua.

\section{Tarea educativa}

Los estudios sociolingüísticos, como el que hemos intentado, tienen una doble virtud:

- Subrayar de diferentes maneras un rasgo fundamental del lenguaje: la armonía de las semejanzas y de las diferencias, la in- 
variante en la variación. Es el lenguaje mismo el que nos propone esta lección de tolerancia.

- Mostrar la configuración de un campo social condicionando el comportamiento lingüístico y en el cual se encuentran eventualmente formas de dominación simbólica. Y esta consideración ofrece elementos de discernimiento social.

De este modo, la reflexión sociolingüística puede hacer un aporte a la tarea educativa, puesto que el aprendizaje de la tolerancia y la introducción a un juicio lúcido del mundo social constituyen objetivos fundamentales del trabajo pedagógico.

En lo que se refiere a la enseñanza del castellano en el contexto multilingüe andino, tres pistas merecerían profundizaciones y aplicaciones:

- Se podría explotar las virtudes didácticas de una trayectoria onomasiológica: a partir de una categoría conceptual, encontrar sus soluciones en Castellano, en Quechua y en Aymara. Esto podría favorecer la comprensión de las dificultades que comporta el paso de una lengua a otra: los alumnos y maestros podrían así comprender el por qué de muchas de sus dificultades.

- En la perspectiva de una valorización del Quechua y del Aymara, y considerando que estas lenguas condicionan la adquisición del Castellano, sería deseable promover un real dominio, oral y escrito, y un desarrollo creativo de estas lenguas andinas.

- Comprendiendo que ciertos hábitos lingüísticos han logrado legitimarse, sea regional o socialmente, y que son plenamente aceptados sobre todo en situación pública, sería igualmente deseable promover el dominio real de tales variantes del castellano. 


\section{BIBLIOGRAFIA}

ALARCOS LLORACH, Emilio

1965 Fonologia española. Gredos. Madrid.

BENVENISTE, Emile

1974 Problèmes de linguistique générale. Gallimard. París.

BOURDIEU, Pierre

.1980 Questions de sociologie. Minuit. París.

CARRION ORDOÑEZ, Enrique

1985 "La lengua española en el ámbito geográfico nacional”, en Boletin de la Academia Peruana de la Lengua. No 20. Lima. pp. 65-86.

CERRON-PALOMINO, Rodolfo

1977 “La motosidad: instrumento de opresión”, en Lingüistica y Educación. Instituto Boliviano de Cultura. La Paz. pp. 129-158.

ESCOBAR, Alberto

1978 Variaciones sociolingüisticas del castellano en el Perú. IEP. Lima.

GODENZZI, Juan Carlos

1985 Variations sociolinguistiques de l'espagnol à Puno-Pérou. Tesis de Tercer Ciclo. Universidad de París IV - Sorbona. París.

1986 Lengua y variación sociolectal: El castellano en Puno. Documento de trabajo del área de Lingüística Andina y Educación. Universidad Nacional del Altiplano. Puno.

KANY, Charles

1976

Sintaxis hispanoamericana. Gredos. Madrid. 
NAVARRO TOMAS, T.

1980 Manual de pronunciación española. CSIC. Madrid.

POTTIER, Bernard

1972 Introduction à l'étude linguistique de l'espagnol. Ediciones hispanoamericanas. París.

1974 Linguistique générale. Klincksieck. París.

1980 "Temps et Espace". Travaux de Linguistique et Litterature, XVIII, 1. Strasbourg.

RODRIGUEZ GARRIDO, José

1982 "Sobre el uso del posesivo redundante en el español del Perú". Lexis vol. VI, No 1. P.U.C. Lima.

SCHMIDELY, Jack

1983 La personne grammaticale et la langue espagnole. Editions Hispaniques. París.

WANDRUSZKA, Mario

1976 Nuestros idiomas: comparables e incomparables, I y II. Gredos. Madrid. 